\title{
Concentration for unknown atomic entangled states via cavity decay
}

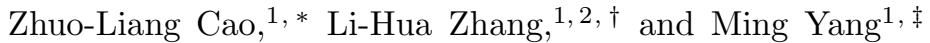 \\ ${ }^{1}$ Schoolof Physics 85 Material Science, Anhui University, Hefei, 230039, People's Republic of China \\ ${ }^{2}$ Department of Physics, Anqing Teacher College, Anqing, 246011, People's Republic of China
}

\begin{abstract}
We present a physical scheme for entanglement concentration of unknown atomic entangled states via cavity decay. In the scheme, the atomic state is used as stationary qubit and photonic state as flying qubit, and a close maximally entangled state can be obtained from pairs of partially entangled states probabilistically.
\end{abstract}

PACS numbers: 03.67.Mn, 03.67.Hk, 03.67.Pp

Entanglement plays an important role [1, 2, 3, 4] in quantum information and computation. To fulfill perfect quantum information processing (QIP) the quantum channel must be maximally entangled usually. But, in the real processing, storage and transmission of quantum states, the maximally entangled states usually collapse into nonmaximally entangled ones or even mixed states because of noise. To achieve faithful QIP, we must convert the nonmaximally entangled states into pure maximally entangled ones. There are many theoretical and experimental schemes that can achieve this conversion, such as entanglement concentration (pure nonmaximally entangled states case) [5], entanglement purification or distillation (mixed entangled states case) [] ].

Recently, Zhao et al. 7] present a practical scheme for entanglement concentration with the polarization beam splitters. For pure nonmaximally photonic polarized entangled states, S. Bose et al. [8] have proposed a scheme, to realize entanglement purification via entanglement swapping. In the above two proposals [7, 8], they utilize photonic state as both stationary qubits and flying qubits. Photonic qubits, despite its perfection as flying qubits, is not ideal for stationary qubits. Oppositely, the atomic states are known as the ideal stationary qubits in quantum information theory. In the realm of QIP, cavity QED is one of the promising candidates dealing with atomic qubits. There are several entanglement concentration and purification schemes for atomic entangled states in cavity QED [9, 10, 11], where the cavity decay is neglected.

However, spontaneous and detected decay are unavoidable in practical quantum information processing [12] in cavity QED system. So, teleportation and generation of atomic entanglement is more general when taking cavity decay into consideration [13, 14, 15, 16, 17]. Obviously, it is possible to generate an entangled state between two atoms, located in two different decay cavities, respectively. Here, we present a scheme for entanglement concentration of unknown atomic entangled states via cavity decay.

\footnotetext{
*Electronic address: zlcao@ahu.edu.cn(Corresponding ${ }^{\sim}$ Author)

${ }^{\dagger}$ Electronic address: zhanglh@aqtc.edu.cn

${ }_{\ddagger}^{\ddagger}$ Electronic address: mingyang@ahu.edu.cn
}

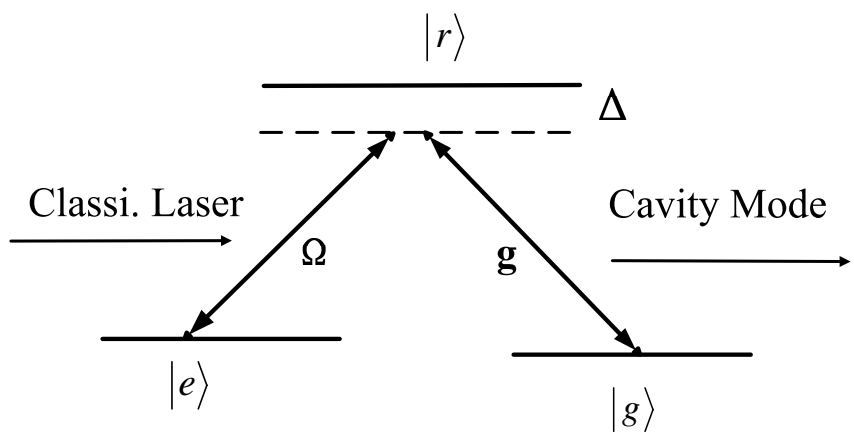

FIG. 1: Level structure of the atoms used in our scheme. The $|e\rangle \rightarrow|r\rangle$ transition is driven by a classical laser pulse with coupling $\Omega$, and the $|r\rangle \rightarrow|g\rangle$ transition is driven by the quantized cavity mode with coupling $g$. Both the classical laser pulse and the cavity mode are detuned from their respective transitions by the same amount $\Delta$.

In this paper, each of the atoms is a three-level system, which has two ground states $|g\rangle,|e\rangle$ (e.g. hyperfine ground states) and an excited state $|r\rangle$ as depicted in Fig. 11 It is an adiabatic evolution for the $|e\rangle \rightarrow|r\rangle$ transition, which is driven by a classical laser pulse with coupling coefficient $\Omega$. The $|r\rangle \rightarrow|g\rangle$ transition is driven by the quantized cavity mode with coupling coefficient $g$. Both the classical laser pulse and the cavity mode are detuned from their respective transitions by the same amount $\Delta$. Assuming the atom is trapped in a specific position in the cavity, and the coupling coefficients $\Omega$ and $g$ are constant during the interaction. In the case of $\Omega g / \Delta^{2}<<1$, the upper level $|r\rangle$ can be decoupled from the evolution. When $\Delta>>\gamma$ the spontaneous decay rate $\gamma$ from $|r\rangle$ level can be neglected [13]. In the interaction picture and suppose $\Omega=g$, the effective Hamiltonian of the atom interacted with the cavity is

$$
H_{e f f}=i \delta\left(a|e\rangle\left\langle g\left|-a^{+}\right| g\right\rangle\langle e|\right)-i k a^{+} a,
$$

where $\delta=g \Omega / \Delta, a$ and $a^{+}$are the annihilation and creation operators of the cavity mode, $k$ is the photon decay rate from the cavity.

In QIP, maximally entangled atomic states are usually distributed among users in different locations. Because of nonperfect generation scheme, the entangled states of the distant atoms may be in the nonmaximally entangled 


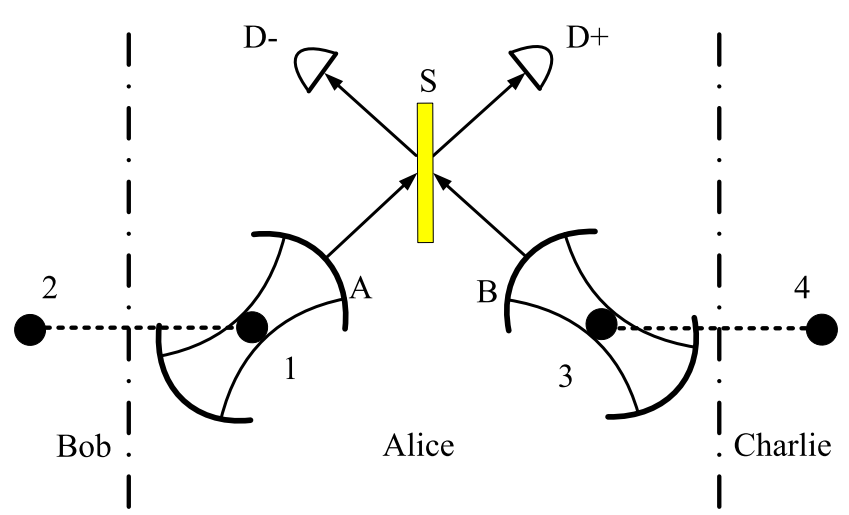

FIG. 2: The setup is adapted to concentrate two nonmaximally entangled pairs into one maximally entangled. Atoms 1 and 3 are trapped in the optical cavities $A$ and $B$, respectively. $S$ is a $50 / 50$ beam splitter and $D_{ \pm}$are single-photon detectors.

states. We can suppose that the entangled states of two pairs atoms

$$
\begin{gathered}
|\Phi\rangle_{12}=a|e\rangle_{1}|g\rangle_{2}+b|g\rangle_{1}|e\rangle_{2}, \\
|\Phi\rangle_{34}=c|e\rangle_{3}|g\rangle_{4}+d|g\rangle_{3}|e\rangle_{4},
\end{gathered}
$$

where $a, b, c$ and $d$ are the normalization coefficients. To extract maximally entangled states from the nonmaximally entangled states, we can use the setup in Fig. 2 Atoms 1 and 3 are trapped in the optical cavities $A$ and
$B$, respectively. Atoms 1 and 3 , cavities $A$ and $B$, beam splitter $S$ and two detectors belong to Alice, atom 2 belongs to Bob, and atom 4 belongs to Charlie. The cavities are prepared in vacuum states initially.

Then, the initial states of system- $A$ (cavity $A$ and atoms 1,2 ) and system- $B$ (cavity $B$ and atoms 3,4 ) are

$$
\begin{aligned}
|\Psi\rangle_{12 A} & =\left(a|e\rangle_{1}|g\rangle_{2}+b|g\rangle_{1}|e\rangle_{2}\right)|0\rangle_{A} \\
|\Psi\rangle_{34 B} & =\left(c|e\rangle_{3}|g\rangle_{4}+d|g\rangle_{3}|e\rangle_{4}\right)|0\rangle_{B} .
\end{aligned}
$$

Alice applies two same classical laser pulses on atoms 1 and 3, respectively, to switch on the effective Hamiltonian $H_{e f f}$ in the System- $A$ and System- $B$ simultaneously. Choosing the interaction time $t_{1}$, which satisfies $\tan \frac{\Omega_{k} t_{1}}{2}=-\frac{\Omega_{k}}{k}\left(\right.$ where $\left.\Omega_{k}=\sqrt{4 \delta^{2}-k^{2}}\right)$, the states of system- $A$ and the system- $B$ evolve into

$$
\begin{aligned}
\left|\Psi^{\prime}\right\rangle_{12 A} & =\frac{\left(a \alpha|g\rangle_{2}|1\rangle_{A}+b|e\rangle_{2}|0\rangle_{A}\right)|g\rangle_{1}}{\sqrt{|a|^{2} \alpha^{2}+|b|^{2}}}, \\
\left|\Psi^{\prime}\right\rangle_{34 B} & =\frac{\left(c \alpha|g\rangle_{4}|1\rangle_{B}+d|e\rangle_{4}|0\rangle_{B}\right)|g\rangle_{3}}{\sqrt{|c|^{2} \alpha^{2}+|d|^{2}}}
\end{aligned}
$$

where $\alpha=-\frac{2 \delta}{\Omega_{k}} e^{-\frac{k t_{1}}{2}} \sin \frac{\Omega_{k} t_{1}}{2}$. The successful probability of evolvement are $P_{A}=\left(|a|^{2} \alpha^{2}+|b|^{2}\right)$ and $P_{B}=\left(|c|^{2} \alpha^{2}+\right.$ $\left.|d|^{2}\right)$, respectively. At the time $t_{1}$, the joint state of atoms 2,4 , and cavity $A, B$ becomes

$$
\left|\Psi\left(t_{1}\right)\right\rangle_{24 A B}=\frac{\left(a \alpha|g\rangle_{2}|1\rangle_{A}+b|e\rangle_{2}|0\rangle_{A}\right) \times\left(c \alpha|g\rangle_{4}|1\rangle_{B}+d|e\rangle_{4}|0\rangle_{B}\right)}{\sqrt{P_{A} P_{B}}}
$$

the success probability of this step is $P_{s u c 1}=P_{A} P_{B}$. If we select $\Omega_{k}>>k, P_{\text {suc } 1} \approx 1$.

Now we consider the detection stage in which we make two single-photon detectors $[13,14]$. Alice will wait for one click at $\mathrm{D}_{+}$or $\mathrm{D}_{-}$for a time interval $t_{2}$, at a time $t_{j}$ in the detection stage $\left(t_{j} \leq t_{2}\right)$, the joint state of atoms 2,4 , and cavities $A, B$ evolves into [18

$$
\left|\Psi\left(t_{j}\right)\right\rangle_{24 A B}=\frac{a \alpha e^{-k t_{j}}|g\rangle_{2}|1\rangle_{A}+b|e\rangle_{2}|0\rangle_{A}}{\sqrt{|a|^{2} \alpha^{2} e^{-2 k t_{j}}+|b|^{2}}} \times \frac{c \alpha e^{-k t_{j}}|g\rangle_{4}|1\rangle_{B}+d|e\rangle_{4}|0\rangle_{B}}{\sqrt{|c|^{2} \alpha^{2} e^{-2 k t_{j}}+|d|^{2}}} .
$$

If only one of the detectors $\mathrm{D}_{ \pm}$clicks, it corresponds to the action of the jump operators $\left(a_{A} \pm a_{B}\right) / \sqrt{2}$ on the joint state $\left|\Psi\left(t_{2}\right)\right\rangle_{24 A B}$, then the joint state of entire system becomes

$$
\left|\Psi\left(t_{2}\right)^{ \pm}\right\rangle_{24 A B}=\frac{a c \alpha e^{-k t_{2}}|g\rangle_{2}|g\rangle_{4}\left(|0\rangle_{A}|1\rangle_{B} \pm|1\rangle_{A}|0\rangle_{B}\right)+\left(a d|g\rangle_{2}|e\rangle_{4} \pm b c|e\rangle_{2}|g\rangle_{4}\right)|0\rangle_{A}|0\rangle_{B}}{\sqrt{|a d|^{2}+|b c|^{2}+2|a c|^{2} \alpha^{2} e^{-2 k t_{2}}}}
$$

If the $\mathrm{D}_{-}$clicked, Alice lets Charlie give $|g\rangle_{4}$ an extra phase shift $\pi$ with respect to $|e\rangle_{4}$ by classical communication channel; if the $\mathrm{D}_{+}$clicked, no extra phase shift is required.

If the initial states of atoms 1,2 and 3,4 satisfy the condition $a=c, b=d$, the state $\left|\Psi\left(t_{2}\right)^{+}\right\rangle_{24 A B}$ will become

$$
\left|\Psi\left(t_{2}\right)^{+}\right\rangle_{24 A B}=\frac{a^{2} \alpha e^{-k t_{2}}|g\rangle_{2}|g\rangle_{4} \frac{1}{\sqrt{2}}\left(|0\rangle_{A}|1\rangle_{B}+|1\rangle_{A}|0\rangle_{B}\right)+a b \frac{1}{\sqrt{2}}\left(|g\rangle_{2}|e\rangle_{4}+|e\rangle_{2}|g\rangle_{4}\right)|0\rangle_{A}|0\rangle_{B}}{\sqrt{|a b|^{2}+|a|^{4} \alpha^{2} e^{-2 k t_{2}}}}
$$


Now, we consider the state $\left|\Psi\left(t_{2}\right)^{+}\right\rangle_{24 A B}$ by tracing over the cavities $A$ and $B$, the reduced density matrix of atoms 2 and 4 is

$$
\rho_{24}=\frac{|a b|^{2}|\Phi\rangle_{2424}\left\langle\left.\Phi|+| a\right|^{4} \alpha^{2} e^{-2 k t_{2}} \mid g\right\rangle_{2}|g\rangle_{44}\left\langle\left. g\right|_{2}\langle g|\right.}{|a b|^{2}+|a|^{4} \alpha^{2} e^{-2 k t_{2}}}
$$

where $|\Phi\rangle_{24}=\frac{1}{\sqrt{2}}\left(|g\rangle_{2}|e\rangle_{4}+|e\rangle_{2}|g\rangle_{4}\right)$, which is the ultimate result of concentration. The total successful probability of obtaining the entangled state in Eq. (9) is $P_{\text {success }}=\left(|a b|^{2}+|a|^{4} \alpha^{2} e^{-2 k t_{2}}\right) \alpha^{2} e^{-2 k t_{2}}\left(1-e^{-2 k t_{2}}\right)$, and the fidelity of the obtained state $|\Phi\rangle_{24}$ is $F=$ $|b|^{2} /\left(|b|^{2}+|a|^{2} \alpha^{2} e^{-2 k t_{2}}\right)$. In the case of $|a / b|<<1$, the fidelity will be $F \approx 1$.

In conclusion, we have proposed an entanglement concentration scheme for unknown atomic entangled states with a finite probability via cavity decay. Compared with other schemes, our scheme has the following advantages: (a) In our scheme, the atomic state is used as stationary qubit and photonic state as flying qubit, which is more feasible experimentally. thus the distant entangled states concentration via high quality fiber will be convenient. (b) Taking cavity decay into consideration, it is more practical to discuss entanglement concentration.

\section{Acknowledgments}

This work is supported by the Natural Science Foundation of the Education Department of Anhui Province under Grant No: 2004kj005zd and Anhui Provincial Natural Science Foundation under Grant No: 03042401 and the Talent Foundation of Anhui University.
[1] C. H. Bennett and S.J. Wiesner, Phys. Rev. Lett. 69, 2881 (1992).

[2] C. H. Bennett, G. Brassard, C. Crépeau, R. Jozsa, A. Peres and W. K. Wootter, Phys. Rev. Lett. 70, 1895 (1993).

[3] A. K. Ekert, Phys. Rev. Lett. 67, 661 (1991).

[4] C. H. Bennett, and D. P. Divincenzo, Nature (London) 404, 247 (2000).

[5] C. H. Bennett, H. J. Bernstein, S. Popescu and B. Schumacher, Phys. Rev. A 53, 2046 (1996).

[6] C. H. Bennett, G. Brassard, S. Popescu, B. Schumacher, J. A. Smoin, \& W. K. Wootters, Phys. Rev. Lett. 76, 722 (1996).

[7] Z. Zhao, T. Yang, Y. A. Chen, A. N. Zhang, and J. W. Pan , Phys. Rev. Lett. 90, 207901 (2003).

[8] S. Bose, V. Vedral, and P. L. Knight, Phys. Rev. A 60, 194 (1999).

[9] M. Yang, Y. Zhao, W. Song, and Z. L. Cao , Phys. Rev.
A 71, 044302 (2005).

[10] Z. L. Cao, and M. Yang, G. C. Guo, Phys. Lett. A 308, 349 (2003).

[11] L. Ye and G. C. Guo, Phys. Lett. A 327, 284 (2004).

[12] M. B. Plenio and P. L. Knight, Phys. Rev. A 53, 2986 (1996).

[13] S. Bose, P. L. Knight, M. B. Plenio, and V. Vedral, Phys. Rev. Lett. 83, 5158 (1999).

[14] X. B. Zou, K. Pahlke, and W. Mathis, Phys. Rev. A 68, $024302(2003)$.

[15] B. Yu, Z.W. Zhou, Y. Zhang, G. Y. Xiang, and G. C. Guo, Phys. Rev. A 70, 014302 (2004).

[16] G. Chimczak, R. Tana., and A. Miranowicz, Phys. Rev. A 71, 032316 (2005).

[17] G. Chimczak, Phys. Rev. A 71, 052305 (2005).

[18] M. B. Plenio, and P. L. Knight, Rev. Mod. Phys. 70, 101 (1998). 